Scientific Paper

\title{
Determination of contributions of scatter and distance error to the source strength of ${ }^{192}$ Ir HDR brachytherapy source
}

\author{
Shwetha BONDEL ${ }^{1, a}$, Manickam RAVIKUMAR ${ }^{1}$ \\ ${ }^{I}$ Department of Radiation Physics, Kidwai Memorial Institute of Oncology, Bangalore - 560029, Karnataka, India \\ ${ }^{a}$ E-mail address: shwetha0828@yahoo.co.in
}

(received 5 April 2016; revised 6 July 2016; accepted 15 July 2016)

\begin{abstract}
High dose rate (HDR) brachytherapy commonly employs a ${ }^{192}$ Ir encapsulated source to deliver high dose to the malignant tissues. Calibrations of brachytherapy sources are performed by the manufacturer using a well-type chamber or by in-air measurement using a cylindrical ionization chamber. Calibration using the latter involves measurements to be carried out at several distances and room scatter can also be determined. The aim of the present study is to estimate the scatter contribution from the walls, floor and various materials in the room in order to determine the reference air kerma rate of an ${ }^{192}$ Ir HDR brachytherapy source by in-air measurements and also to evaluate the error in the setup distance between the source centre and chamber centre. Air kerma measurements were performed at multiple distances from $10 \mathrm{~cm}$ to $40 \mathrm{~cm}$ between the source and chamber. The room scatter correction factor was determined using the iterative technique. The distance error of $-0.094 \mathrm{~cm}$ and $-0.112 \mathrm{~cm}$ was observed for chamber with and without buildup cap respectively. The scatter component ranges from $0.3 \%$ to $5.4 \%$ for the chamber with buildup cap and $0.3 \%$ to $4.6 \%$ without buildup cap for distances between 10 to $40 \mathrm{~cm}$ respectively. Since the average of the results at multiple distances is considered to obtain the actual air kerma rate of the HDR source, the seven distance method and iterative technique are very effective in determining the scatter contribution and the error in the distance measurements.
\end{abstract}

Key words: in-air calibration; room scatter; air kerma rate; distance error; multiple distance.

\section{Introduction}

Most hospitals prefer calibrating the ${ }^{192}$ Ir HDR brachytherapy source using a well-type chamber as it is cumbersome to do the calibration using a Farmer chamber with a calibration jig since it involves acquiring the data by setting the source to chamber at different, accurately measured distances, and also obtaining the room scatter. Generally, a Farmer type ionization chamber calibrated at NIST or ADCL has better calibration factor accuracy than that of a well chamber [1]. Thus, more accurate calibration results could be potentially obtained by using the Farmer chamber than by using the well chamber. The recommended quantity by National Institute of Standards and Technology (NIST) for specifying the source strength for photon emitting brachytherapy sources is the air kerma strength (AKS) $[2,3] \mathrm{K}(\mathrm{d})$, which is defined as the product of air kerma rate at a calibration distance, $d$, in the free space, measured along the transverse bisector of the source, and the square of the distance, $d$. The uncertainty in the determination of AKS is estimated to be $\pm 5 \%$ [3]. An error in the estimation of source strength introduces systematic errors in the dose determination for patients treated with the source. This necessitates the error to be kept as low as possible and the accurate determination of source strength of ${ }^{192}$ Ir HDR brachytherapy source. The in-air measurement of source strength using cylindrical ionization chamber requires the determination of scatter radiation from walls and floor of the room and also the offset in positioning of the chamber.

Few studies [4-10] have been reported for determining the room scatter contribution from an ${ }^{192}$ Ir HDR brachytherapy source. Goestch et al [4] described a method for calibrating ${ }^{192}$ Ir HDR brachytherapy afterloading systems. Procedures are described for determining the room scatter and source to chamber distance corrections using multiple distance method. Also a total (wall+cap) thickness of $0.3 \mathrm{~g} / \mathrm{cm}^{2}$ is recommended to assure charged particle equilibrium and to exclude secondary electrons emitted from the source encapsulation. Stump et al [5] used the in-air technique with seven distance method for calibrating two new ${ }^{192} \mathrm{Ir}$ sources. The overall uncertainty of the measurement was determined by performing an uncertainty budget. Rasmussen et al [6] compared the interim air-kerma strength standard established by Goetsch et al [4] for HDR ${ }^{192} \mathrm{Ir}$ brachytherapy sources with measurements of the various source models using modified techniques from the literature. They found the differences between the source models to be statistically significant but confirmed that it is not necessary to update the current interim standard of seven distance method.

In the present study the scatter contribution from the walls, floor and various materials in the room and offset in the measurement of actual source centre to chamber centre distance has been determined. 


\section{Materials and Methods}

The Farmer type ionization chamber of $0.65 \mathrm{cc}$ volume (FC-65) and Dose1 electrometer (Scanditronix/Wellhofer) were used in the experiment. The chamber has a poly oxy methylene (POM) buildup cap (thickness $=0.55 \mathrm{~g} / \mathrm{cm}^{2}$ and density $=1.4 \mathrm{~g} / \mathrm{cm}^{3}$ ), outer electrode made of graphite (density $=1.8 \mathrm{~g} / \mathrm{cm}^{3}$ ), inner electrode of Aluminium with a total length of $2.05 \mathrm{~cm}$ and a diameter of $1.0 \mathrm{~mm}$. The chamber operates at a maximum polarizing voltage of $\pm 300 \mathrm{~V}$. The applicator used is a needle made of stainless steel 1.4401 (AISI 316) with an outer diameter of $1.65 \mathrm{~mm}$ and wall thickness of $0.15 \mathrm{~mm}$. The Gammamed Plus ${ }^{192} \mathrm{Ir}$ source is $4.52 \mathrm{~mm}$ long with an active length of $3.5 \mathrm{~mm}$. The source has a stainless steel encapsulation with an outer diameter of $0.9 \mathrm{~mm}$ and active diameter of $0.6 \mathrm{~mm}$.

An indigenously designed jig made of non-scattering plastic material (rigid Poly Vinyl Chloride foam board) which has provisions to hold the chamber and fix the source applicator at various distances from the chamber was locally fabricated for the experiment. The experimental setup is shown in Figure 1. The multiple distance (seven-distance) method as recommended by IAEA [11] was followed to perform the scatter measurements. The jig was placed at the centre of the room at least $1 \mathrm{~m}$ above the floor and at $1 \mathrm{~m}$ distance from any of the walls. The chamber with the build-up cap was fixed at one end of the jig while the applicator needle was moved to different distances $(10,15,20,25,30,35$ and $40 \mathrm{~cm})$ from the chamber. Initially, electrometer readings were noted by moving the source to various dwell positions (step size of $2.5 \mathrm{~mm}$ ) vertically along the applicator to determine the dwell position showing maximum response. Subsequently for all the measurements, the source was made to dwell in the maximum response position. The measured charge for a time interval $t$ (sec) was corrected for ambient temperature and pressure, air attenuation, ion recombination, non uniform electron fluence within the air cavity [11-13] and applicator attenuation. No correction for transit time was made as the electrometer readings were obtained in the current mode. The entire procedure was repeated using chamber without the build-up cap.

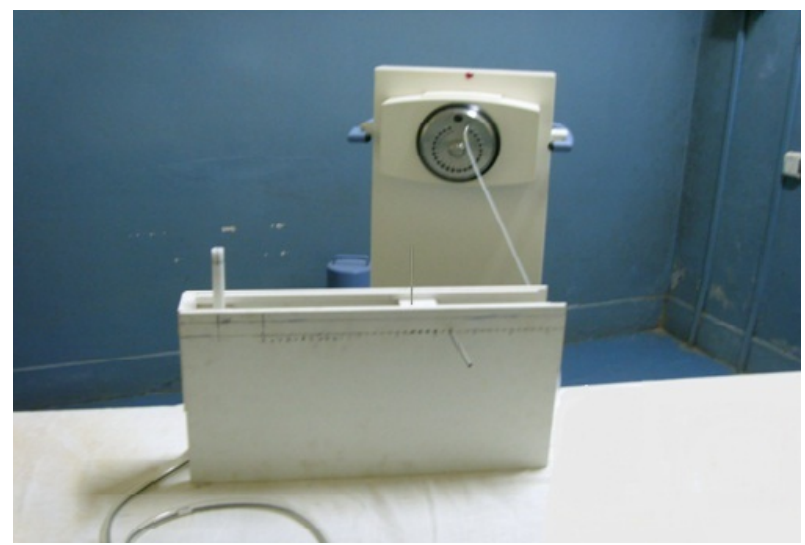

Figure 1. Experimental setup for the Reference Air Kerma Rate measurements.
The meter reading obtained at any distance $\mathrm{d}$ includes contribution from primary radiation $\left(\mathrm{M}_{\mathrm{p}}\right)$ and scatter radiation $\left(\mathrm{M}_{\mathrm{s}}\right)$ which is expressed as:

$M_{d}=M_{p}+M_{s}$

where the primary component $\mathrm{M}_{\mathrm{p}}$ follows the inverse square law and $M_{s}$ is assumed to be constant within the range of measured distances with the assumption that same amount of scattered photons reach the chamber irrespective of the distance $d$. Hence, $M_{p}$ can be written in the form of the following equation:

$M_{p}=\left(M_{d}-M_{s}\right)=f /\left(d^{1}\right)^{2}$

Eq. 2

which is rewritten as:

$f=\left(M_{d}-M_{s}\right)(d+c)^{2}$

where $d^{1}=(d+c)^{2}$, $f$ is a proportionality constant, $d$ is the nominal distance between the centre of source and centre of chamber and $\mathrm{c}$ is the error in the distance measurement.

A set of three equations of the form of Equation 3 at three nominal distances were used to solve for the three unknowns $\mathrm{M}_{\mathrm{s}}$, c and $\mathrm{f}$. Making different combinations of three such equations at the 7 measured distances produces 35 solutions of the unknown variables. The equations were solved using Microsoft Excel worksheet. The average of these 35 solutions was used to determine the values of $\mathrm{M}_{\mathrm{s}}$ and $\mathrm{c}$. Knowing the values of $\mathrm{M}_{\mathrm{s}}$ and $\mathrm{c}$, $\mathrm{f}$ was calculated from Equation 3 at each distance. The results obtained were also verified using an independent software program (Mathematica 7). The reference air kerma rate (RAKR) is calculated according to the Deutsche Gesellschaft fur Medizinische Physik (DGMP) recommendations $[14,15]$ from the average $f$ value at the 7 distances as follows:

$\left(K_{a}\right)_{a}=N_{D W}\left(\frac{1}{1-g_{a}}\right)\left(\mu_{e n} / \rho\right)_{a-w} k_{Q} k_{r} k_{A K} A_{w} f$

Eq. 4

where

- $\mathrm{N}_{\mathrm{DW}}$ is the absorbed dose to water calibration factor of the ionization chamber for ${ }^{60} \mathrm{Co}$ beam.

- $\mathrm{g}_{\mathrm{a}}$ is the fraction of energy of the secondary electrons, which is lost in bremsstrahlung. The $g_{a}$ value of 0.001 given by DGMP task group [16] is used in this study.

- $\left(\mu_{\mathrm{en}} / \rho\right)_{\mathrm{a}-\mathrm{w}}$ is the ratio of mass energy absorption co-efficient of air to that of water. The value of $\left(\mu_{\mathrm{en}} / \rho\right)_{\mathrm{a}-\mathrm{w}}$ for ${ }^{192} \operatorname{Ir}$ source used in this study is 0.899 .

- $\mathrm{k}_{\mathrm{Q}}$ is the beam quality correction factor, which accounts for the differences in the energy spectrum of the reference photon beam (usually ${ }^{60} \mathrm{Co}$ ) for which the chamber has been calibrated and ${ }^{192}$ Ir spectrum (generally determined through interpolation of calibration factor of ${ }^{60} \mathrm{Co}$ and highest X-ray energy available). Since the energy dependence of modern thimble chambers is marginal [16] a value of 1.0 is used.

$\cdot \mathrm{k}_{\mathrm{r}}=\left(1 / \mathrm{r}_{0}\right)^{2}$, where $\mathrm{r}_{0}$ is the reference distance of 1 meter.

- $\mathrm{k}_{\mathrm{AK}}$ is the correction factor for application of ${ }^{60} \mathrm{Co}$ build-up cap. A value $\mathrm{k}_{\mathrm{AK}}=1.005$ is recommended [14]. 
- $A_{w}$ is the chamber wall correction factor [17] to account for scattering and attenuation in the wall of the ionization chamber. $\mathrm{A}_{\mathrm{w}}$ is determined using a formula $\mathrm{A}_{\mathrm{w}} \approx 1-\gamma \mathrm{t}$, where $\gamma$ is the attenuation and scattering fraction per wall thickness $\left(\mathrm{cm}^{2} / \mathrm{g}\right)$ and taken as 0.0277 and $\mathrm{t}$ is the total thickness $\left(\mathrm{g} / \mathrm{cm}^{2}\right)$ of wall material.

The recombination correction factor $\mathrm{k}_{\mathrm{s}}$ was derived using the following equation:

$k_{S}=\frac{\left(V_{1} / V_{2}\right)^{2}-1}{\left(V_{1} / V_{2}\right)^{2}-\left(M_{1} / M_{2}\right)}$

where $M_{1}$ and $M_{2}$ are the meter readings at the voltages $V_{1}$ and $\mathrm{V}_{2}$ respectively and $\mathrm{V}_{1} / \mathrm{V}_{2} \geq 3[18]$.

\section{Results}

Table 1 shows the average values of $\mathrm{f}, \mathrm{c}, \mathrm{M}_{\mathrm{s}}$ and RAKR obtained using ionization chamber with and without build-up cap. The $\mathrm{c}$ value obtained from iterative method was $-0.112 \mathrm{~cm}$ which shows that the centre of the source was actually about $1.12 \mathrm{~mm}$ closer to the centre of the chamber with the build-up cap. In the absence of build-up cap the c value obtained was $-0.115 \mathrm{~cm}$. The room scatter contribution $\left(\mathrm{M}_{\mathrm{s}}\right)$ is expressed as the percentage of primary component of meter reading at the measured distances and the variation of $\mathbf{M}_{\mathrm{s}}$ with distance is shown in Figure 2. The scatter component ranges from $0.3 \%$ to $5.4 \%$ for the chamber with buildup cap and $0.3 \%$ to $4.6 \%$ without buildup cap for distances between 10 and $40 \mathrm{~cm}$. There is no considerable change in the percentage of scatter between chamber with and without buildup cap.

The percentage deviation of measured RAKR from the manufacturer quoted value was $-0.94 \%$ and $2.5 \%$ with and without build-up cap respectively. The influence of the scatter and distance correction factors on the source strength was evaluated by calculating the RAKR without including these two factors. In that case, a variation of $2.56 \%$ and $5.95 \%$ from the manufacturer specified value is observed in the air kerma measurements using chamber with and without build-up cap respectively.

Figure 3 shows the variation of $\mathrm{f}=\mathrm{M}_{\mathrm{p}}\left(\mathrm{d}^{1}\right)^{2}$ and the products $M_{d} d^{2}, M_{p} d^{2}$ and $M_{d}\left(d^{1}\right)^{2}$ with distance. Here $f$ is corrected for both scatter and distance error while, the product $\mathrm{M}_{\mathrm{p}} \mathrm{d}^{2}$ is corrected for scatter but not corrected for distance error, $M_{d}\left(d^{1}\right)^{2}$ is corrected for distance error but not corrected for scatter and $\mathrm{M}_{\mathrm{d}} \mathrm{d}^{2}$ is not corrected for both scatter and distance error. From the figure we see that the points representing $f$ almost lie on a straight line indicating that $f$ is independent of changes in distance. Hence $f$ satisfies the inverse square law which is a necessary criteria for source calibration [4].

Figure 4 shows the graph of ratio of ionization of the chamber without buildup cap to with buildup cap vs the source to chamber distance. A gradual reduction in ionization from $2.4 \%$ to $2.0 \%$ was observed from $10 \mathrm{~cm}$ to $25 \mathrm{~cm}$ source to chamber distance. A further reduction up to $1.65 \%$ is seen for distance of $40 \mathrm{~cm}$. The excess ionization produced due to the absence of build-up cap is mainly due to the detection of secondary electrons emitted from the source encapsulation especially at shorter source to chamber distances. Though here the secondary electrons produced due to the presence of buildup cap is missing, the detected secondary electrons from the source encapsulation supersedes that produced from the buildup cap.

Table 1. Calculated average values (with standard deviation) of $f$, c, $M_{s}$ and percentage variation in RAKR using ionization chamber with and without build-up cap for six measurements

\begin{tabular}{ccc}
\hline \hline Parameter & With Build-up cap & Without Build-up cap \\
\hline $\mathrm{c}(\mathrm{cm})$ & $-0.112 \pm 0.018$ & $-0.115 \pm 0.018$ \\
$\mathrm{M}_{\mathrm{s}}(\mathrm{nc})$ & $0.0052 \pm 0.001$ & $0.0049 \pm 0.002$ \\
$\mathrm{f}\left(\mathrm{nC} \mathrm{cm}^{2}\right)$ & $157.26 \pm 5.10$ & $161.05 \pm 5.26$ \\
$\mathrm{RAKR}$ & $-0.94 \pm 0.42$ & $2.5 \pm 0.53$ \\
\hline \hline
\end{tabular}

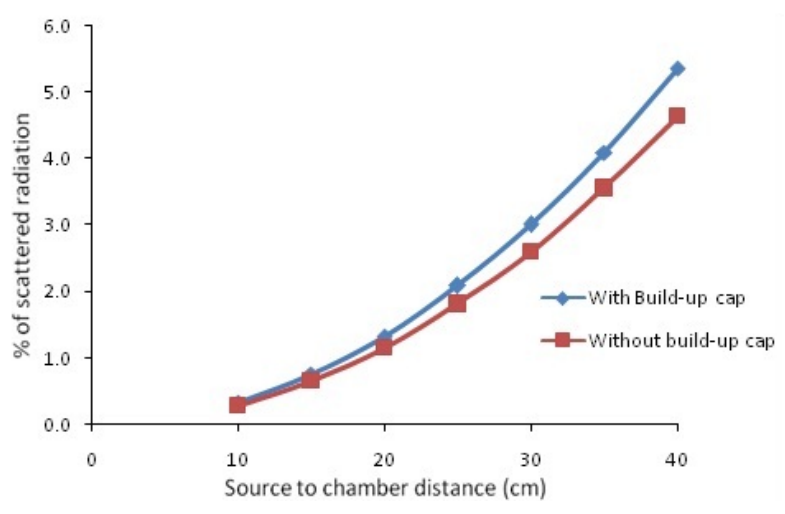

Figure 2. Variation of percentage of scatter contribution with the nominal source to chamber distance.

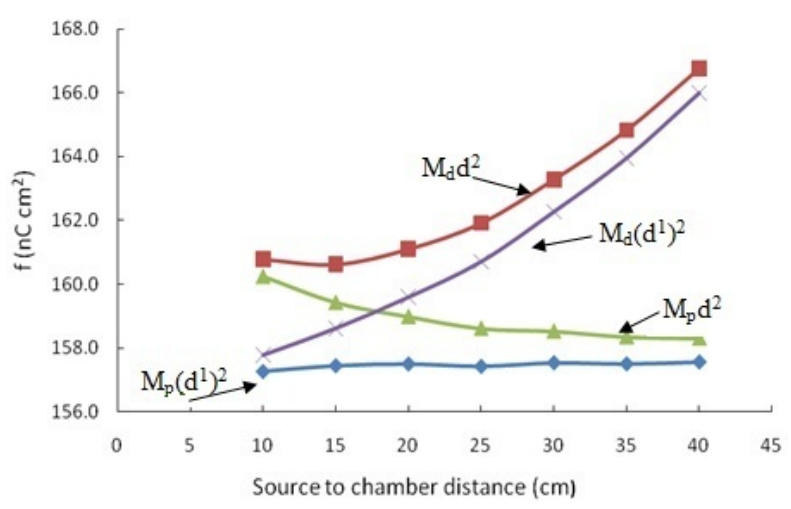

Figure 3. Dependence of $f$ and other products $M_{d} d^{2}, M_{p} d^{2}$ and $M_{d}\left(d^{1}\right)^{2}$ with nominal source to chamber distance.

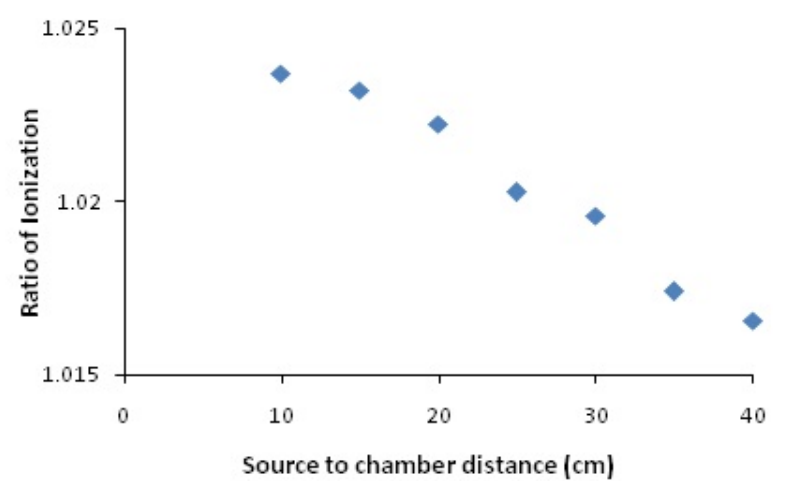

Figure 4. Ratio of ionization without buildup cap to with buildup cap vs. nominal source to chamber distance. 


\section{Discussion}

In the present study we have evaluated the contribution of scatter radiation from a HDR brachytherapy room to the source calibration by in-air measurement. Also the error in positioning the source with respect to the chamber was evaluated. The meter reading observed was corrected for ambient conditions, ion recombination, air attenuation, non uniformity of electron fluence and applicator attenuation. Considering stainless steel AISI 316 to be equivalent to ANSI 303/304 the value of effective attenuation factor was taken as $0.030 \pm 0.002[9,16]$. Hence, a correction factor of 1.0045 was used to correct for the attenuation of the metallic applicator. The correction factor for air attenuation was taken from IAEA Tecdoc 1274 [11] and the non-uniformity correction factor was calculated using Bielajew anisotropic theory [13]. The ion recombination correction was calculated by taking readings at two different voltages $(300 \mathrm{~V}$ and $100 \mathrm{~V})$. In the iterative method three equations corresponding to three different distances were solved at a time. The values of $\mathrm{c}$ were substituted in the equations by trial and error process. The particular value of $\mathrm{c}$ which resulted in the convergence of $M_{s}$ value was taken as the solution. Hence 35 solutions of $\mathrm{c}$ and $\mathrm{M}_{\mathrm{s}}$ were obtained by different combinations of the 7 equations. Then $f$ value was determined from the average of $\mathrm{c}$ and $\mathrm{M}_{\mathrm{s}}$ values from Equation 3 .

An uncertainty exists in the position of the source within the metallic applicator. The inner diameter of the dosimetry applicator is $1.35 \mathrm{~mm}$ and the outer diameter of the ${ }^{192} \mathrm{Ir}$ source is $0.9 \mathrm{~mm}$. Hence the source can displace a maximum of \pm 0.22 $\mathrm{mm}$ with respect to the central axis of the applicator. The uncertainty in the determination of calibration factor $\left(\mathrm{N}_{\mathrm{DW}}\right)$ reported by the secondary standard dosimetry laboratory (SSDL) is $1.5 \%$ at $95 \%$ confidence level $(2 \sigma)$ for $0.65 \mathrm{cc}$ chamber with Dose1 electrometer. The charge leakage associated is less than $0.15 \%$ for $0.65 \mathrm{cc}$ chamber.

\section{Conclusion}

Room scatter measurements can be performed with good accuracy using a specially designed jig which contributes minimum scatter radiation. The iterative method though is time consuming; it is a simple and easy method for evaluating the unknown parameters with the aid of equations corresponding to various distances. The scatter contribution and the distance error can be determined accurately using this method since these parameters are the results of multiple solutions obtained from measurements performed at seven different distances.

\section{Acknowledgements}

The authors would like to thank Mr. T Vijaya Reddy, Mould Room Technician, KMIO for fabricating the jig, Prof. S.L. Keshava, Retired Professor, KMIO and Dr. V Shanmugam, Department of Biostatistics, NIMHANS for their help and support in solving the mathematical equations.

\section{References}

[1] Chang L, Ho SY, Chui CS, et al. Room scatter factor modelling and measurement error analysis of 192Ir HDR calibration by a Farmer chamber. Phys Med Biol. 2007;52(3):871-877.

[2] AAPM. Specification of brachytherapy source strength. New York, NY: American Association of Physicists in Medicine, AAPM Report 21; 1987.

[3] Nath R, Anderson LL, Luxton G, et al. Dosimetry of interstitial brachytherapy sources. Recommendations of the AAPM Radiation Therapy Committee Task Group No. 43. Med Phys. 1995;22(2):209-234.

[4] Goetsch SJ, Attix FH, Pearson DW, et al. Calibration of 192Ir high dose rate afterloading systems. Med Phys. 1991;18(3):462-467.

[5] Stump KE, DeWerd LA, Micka JA, et al. Calibration of new high dose rate 192Ir sources. Med Phys. 2002;29(7):1483-1488.

[6] Rasmussen BE, Davis SD, Schmidt CR, et al. Comparison of air-kerma strength determinations for HDR 192Ir sources. Med Phys. 2011;38(12):6721-6729.

[7] Palani Selvam T, Govinda Rajan KN, Nagarajan PS, et al. Monte Carlo aided room scatter studies in the primary air kerma strength standardization of a remote afterloading 192Ir HDR source. Phys Med Biol. 2001;46(9):2299-2315.

[8] Patel NP, Majumdar B, Vijayan V. Study of scattered radiation for in-air calibration by a multiple-distance method using ionization chambers and an HDR 192Ir brachytherapy source. Br J Radiol. 2006;79(940):347-352.

[9] Patel NP, Majumdar B, Vijiyan V, et al. In-air calibration of an HDR 192Ir brachytherapy source using therapy ion chambers. J Cancer Res Ther. 2005;1(4):213-220.

[10] Kumar S, Srinivasan P, Sharma SD, et al. Evaluation of scatter contribution and distance error by iterative methods for strength determination of HDR 192Ir brachytherapy source. Med Dos. 2010;35(3):230-237.

[11] IAEA. Calibration of photon and beta ray sources used in brachytherapy - Guidelines on standardized procedures at Secondary Standards Dosimetry Laboratories (SSDLs) and hospitals. Vienna: International Atomic Energy Agency, IAEA-TECDOC-1274; 2002.

[12] Kondo S, Randolph ML. Effect of finite size of ionization chambers on measurements of small photon sources. Rad Res. 1960;13(1):37-60. 
[13] Bielajew AF. Correction factors for thick-walled ionization chambers in point source photon beams. Phys Med Biol. 1990;35(4):501516.

[14] Deutsche Gesellschaft für Medizinische Physik e.V. Praktische Dosimetrie in der HDR-Brachytherapie. DGMP-Bericht 13. 1999.

[15] Deutsches Institut fuer Normung. DIN 6809, Teil 2, Klinische Dosimetrie, Brachytherapie mit umschlossenen gammastrahlenden radioaktiven Stoffen. Berlin: Beuth Verlag; 1993.

[16] Baltas D, Geramani K, Ioannidis GT, et al. Comparison of calibration procedures for 192Ir high-dose-rate brachytherapy sources. Int J Radiat Oncol Biol Phys. 1999;43(3):653-661.

[17] Marechal MH, de Almeida CE, Ferreira IH, et al. Experimental derivation of wall correction factors for ionization chambers used in high dose rate 192Ir source calibration. Med Phys. 2002;29(1):1-5.

[18] IAEA. Absorbed Dose Determination in Photon and Electron Beams, An International Code of Practice. Vienna: International Atomic Energy Agency, Technical Reports Series No. 277 (2nd Edition); 1987. 